\title{
Mitigation of Flow-Induced Vibration of Steel Catenary Risers with Pounding Tuned Mass Damper
}

\author{
Peng Zhang $\mathbb{D}^{1,2}$ Haisu Wang $\mathbb{D}^{1},{ }^{1}$ Jiashu Liu $\mathbb{D}^{1},{ }^{1}$ Hailong Liu $\mathbb{D}^{1},{ }^{1}$ and Chunyi Cui $\mathbb{D}^{1}$ \\ ${ }^{1}$ Institute of Road and Bridge Engineering, Dalian Maritime University, Dalian 116023, China \\ ${ }^{2}$ State Key Laboratory of Coastal and Offshore Engineering, Dalian University of Technology, Dalian116023, China \\ Correspondence should be addressed to Chunyi Cui; cuichunyi@dlmu.edu.cn
}

Received 18 June 2021; Accepted 20 January 2022; Published 18 February 2022

Academic Editor: Alvaro Cunha

Copyright (c) 2022 Peng Zhang et al. This is an open access article distributed under the Creative Commons Attribution License, which permits unrestricted use, distribution, and reproduction in any medium, provided the original work is properly cited.

Steel catenary risers (SCR) have been widely used in the offshore oil industry. This paper proposes using a novel vibration control device, i.e., the pounding tuned mass damper (PTMD), to mitigate the flow-induced vibration of an SCR. The numerical model of the SCR and the PTMD has been established in ANSYS. The soil-pipe interaction is simulated by nonlinear springs based on $p-y$ curves. A single degree of freedom structure is utilized to verify the PTMD model established in ANSYS using APDL. Afterwards, numerical studies are carried out to validate the vibration control performance of the proposed PTMD and to investigate the influence of mass ratio and the location of the PTMD. The numerical results imply that the PTMD is very effective in reducing the displacement of the SCR under different oceanic conditions. The vibration control effectiveness will increase with the increase in mass ratio. The maximum vibration reduction is achieved when the PTMD is located at the place where the largest amount of motion takes place.

\section{Introduction}

The past decades have seen a massive construction of marine structures, including offshore turbines [1], platforms, and subsea risers for exploring ocean resources. Of all these structures, steel catenary risers (SCRs), which are often pipeline structures with a large slender ratio [2, 3], have been widely used in engineering practice to connect subsea production well and offshore platforms, as well as to convey production chemicals and hydrocarbons in oil and gas exploration.

The SCR is a typical high flexible structure with a long span and low damping, making it susceptible to oscillations induced by a variety of internal and external sources [4]. During the past decades, extensive studies have been carried out to investigate the behavior of an SCR under vortexinduced vibration [5], internal slug flow-induced vibration [6], external flow-induced vibration [7], and vibration caused by the motion of the floating platform or other types of oil vessel $[8,9]$, among others. These undesired vibrations are the main reason for the damage or fatigue of an SCR. Consequently, a number of vibration control techniques or devices have been developed to dampen the SCR so as to improve its safety and elongate its service life.

Vibration techniques generally fall into two categories: active control and passive control. The active control methods employ sensors and actuators to provide a controlling force to restrain the motion of a riser. Early in the 1990s, Lecordier et al. [10] proposed increasing the viscosity of the fluid by heating the surface of the pipe. Artana et al. [11] proposed an electrohydrodynamic actuator and validated its control effectiveness via an experimental study. Wang et al. [12] proposed a rotating wing system to interfere with the vortex shedding of risers, thus reducing their vibration. Numerical results demonstrated that the amplitude ratio was decreased by $99.2 \%$ (from 0.98 to 0.008 ). Recently, the concept of boundary control has been proposed and widely studied. The boundary control employs active controllers at the top end of the riser, providing control force based on some algorithm [13-15]. Liu and Guo [16] designed a feedback boundary controller for the vessel-riser system. A high-gain observer was proposed to estimate the unmeasurable state of system. A numerical simulation revealed the effectiveness and stability of the controller. Afterward, an 
adaptive controller was developed to achieve robust control of the riser system [17].

Although many active control methodologies have been developed and verified numerically and experimentally, their feasibility still needs to be validated in a harsh marine environment. On the other hand, passive vibration control techniques are also widely studied and accepted for engineering practice. The passive control techniques most often aim to modify the geometry of the riser and thus reduce the external force added to the pipe. Typical devices include helical strakes [18-20], shrouds [21, 22], fairings [23-25], and splitters [26-29]. Recently, Matin Nikoo et al. proposed a novel system called the pipe-in-pipe system [30-32] to reduce the vibration of subsea pipelines. The pipe-in-pipe system added a specially designed spring and dashpot between the inner pipe and the outside pipe. Both numerical and experimental results have verified the effectiveness of this system.

In previous studies, the pounding tuned mass damper (PTMD) has been proposed for vibration control of a variety of structures [33], including subsea jumpers [34-38], bridge cables [39], traffic signal poles [40, 41], building pipelines [42], and other slender structures. The numerical results and experimental results verified the effectiveness and robustness of the PTMD. Therefore, this paper aims to dampen the flow-induced vibration of an SCR with the PTMD. The remaining part of the paper is structured as follows: in Section 2, the finite element model of the SCR and the method to simulate structure-soil interaction will be presented, in Section 3, the mechanism and numerical model of the PTMD will be presented and validated, in Section 4, vibration control effectiveness and the influence of key design parameters will be discussed, and in Section 5, concluding remarks will be summarized to close the paper.

\section{Numerical Model of the SCR}

2.1. FE Model of the SCR. In order to study the vibration control performance of the proposed PTMD, the steel catenary riser reported in [43] is selected as the major structure to be controlled. As illustrated in Figure 1, the riser is made of X65 steel pipes, with an outside diameter of $533 \mathrm{~mm}$ and a thickness of $50 \mathrm{~mm}$. The $119 \mathrm{~m} \mathrm{SCR}$ is composed of 3 segments: one segment is a $70 \mathrm{~m}$ long pipe horizontally lying on the seabed. Another segment is a $35 \mathrm{~m}$ pipe hanging vertically from an offshore platform. The third segment is a curved pipe with a radius of $10 \mathrm{~m}$, connecting the horizontal segment and the vertical segment.

In this paper, a finite element (FE) model of the SCR is established in ANSYS using the embedded APDL language. The SCR is made of X65 type steel. The mass density, elastic module, and Poisson's ratio of this material are $7.85 \times 10^{3}$ $\mathrm{kg} / \mathrm{m}^{3}, 206 \mathrm{GPa}$, and 0.3 , respectively. The SCR is discreted into 120 sections, and each section is modeled by a PIPE59 element, as shown in Figure 1. Furthermore, the interaction between the pipe and the surrounding soil is simulated by the COMBIN39 element. Pipe soil interaction is generally represented by the $p-y$ curve. This element can be defined by two nodes and a generalized load-deformation curve. The $p$ $y$ relationship is a force-displacement relationship in essence, so the parameters of the $p-y$ curve can be input into the COMBIN39 element to simulate the pipe soil interaction in the ocean. The first six order frequencies are listed in Table 1, and the first six order modal shapes can be found in Figure 2.

2.2. Simulation of the Riser-Seabed Interaction. Interaction between the structure foundation and surrounding soils can have a large influence on the dynamic response of the structure [44-46]. The SCR often has a long section laying on the seabed. The interaction of the soil and the steel pipes may have a great influence on the behavior of the SCR and, consequently, has been extensively studied by many researchers, both theoretically and experimentally [47], with a variety of mathematical models developed to characterize the behavior of pipe and the soil. Of all these models, the nonlinear spring model based on $p-y$ curves is characterized by its simplicity and high computation efficiency and is thus applied in this study.

In this study, the horizontal section of the steel riser was modeled by PIPE59 elements and the seabed soil was simulated by COMBIN39 elements, with the strength of the soil determined by the empirical equation proposed by Aubeny et al. [48].

$$
P=N_{P} S_{U} D
$$

Here, $P$ is the strength of the seabed soil, $S_{U}$ is the undrained shear strength, $N_{P}$ is a strength coefficient determined by the embedded depth, and the diameter of the pipe, $D$.

2.3. Flow-Induced Vibration of the SCR. During the prolonged service life of an SCR, it may encounter significantly varied oceanic conditions. Consequently, the proposed PTMD is expected to exhibit satisfying vibration suppression efficiency under most situations. In this study, therefore, five different oceanic conditions (i.e., OC1 to OC5) are defined as listed in Table 2.

Based on the literature [49], in the $x$ and $z$ direction, the motion of seawater particles is as follows:

$$
\begin{aligned}
& u_{x}(x, z, t)=\sum_{n=1}^{\infty} \frac{g A_{n} k_{n}}{w_{n}} \frac{\cosh \left(k_{n}(z+d)\right)}{\cosh \left(k_{n} d\right)} \cos \left(k_{n} x-w_{n} t+\varepsilon_{n}\right) \\
& u_{z}(x, z, t)=\sum_{n=1}^{\infty} \frac{g A_{n} k_{n}}{w_{n}} \frac{\cosh \left(k_{n}(z+d)\right)}{\cosh \left(k_{n} d\right)} \sin \left(k_{n} x-w_{n} t+\varepsilon_{n}\right),
\end{aligned}
$$

where $u_{x}(x, z, t)$ and $u_{z}(x, z, t)$ are the velocity of the water particle in the $x$ and $z$-direction. $A_{n}, \omega_{n}, \varepsilon_{n}$ are the amplitude, circular frequency, and phase of the wave. $d$ and $g$ denote the depth of water and gravity acceleration.

After derivation of (2) and (3), in the $x$ and $z$ direction, the acceleration of the seawater particle is attained as follows: 


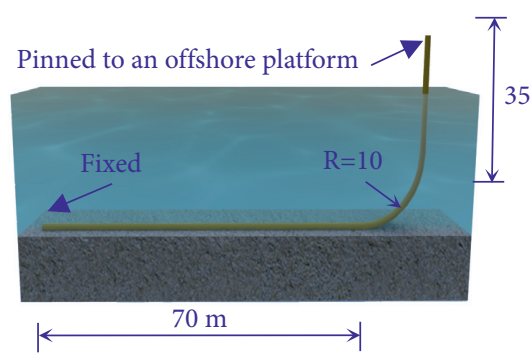

(a)

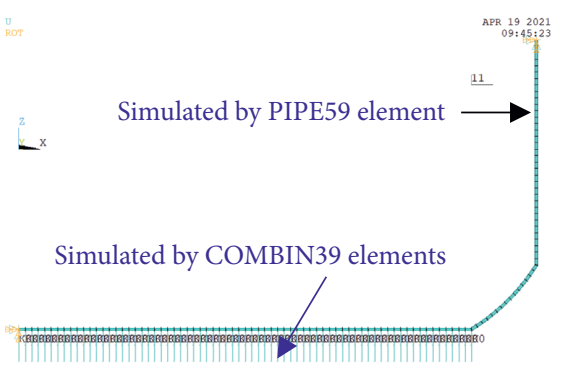

(b)

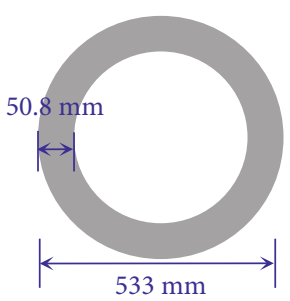

(c)

Figure 1: : Model of the SCR: (a) configuration, (b) FE model, and (c) cross-section.

TABLE 1: : Frequency of the SCR.

\begin{tabular}{lcccccc}
\hline Modal order & $1^{\text {st }}$ & $2^{\text {nd }}$ & $3^{\text {rd }}$ & $4^{\text {th }}$ & $5^{\text {th }}$ & $6^{\text {th }}$ \\
\hline Frequency $(\mathrm{Hz})$ & 0.187 & 0.445 & 0.511 & 1.087 & 1.120 & 1.725 \\
\hline
\end{tabular}

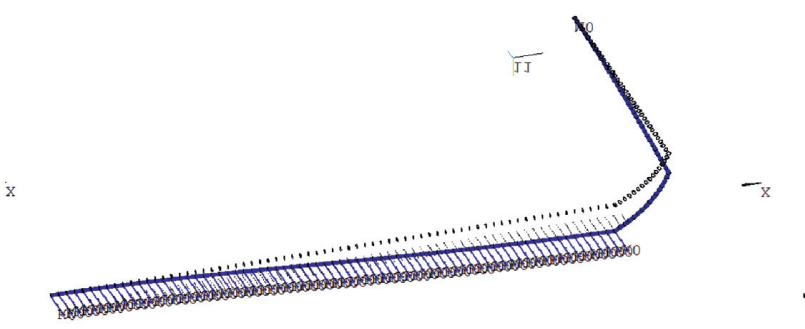

(a)



(c)

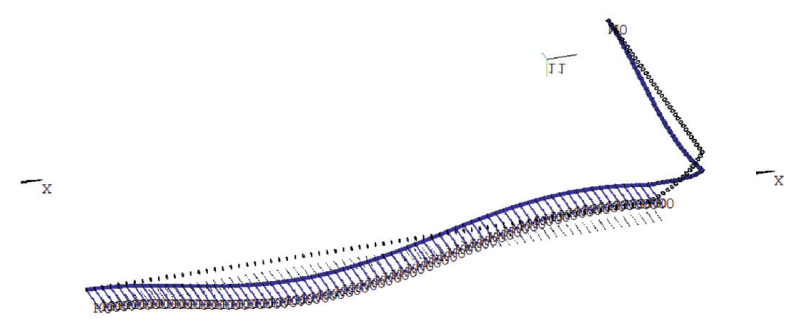

(e)

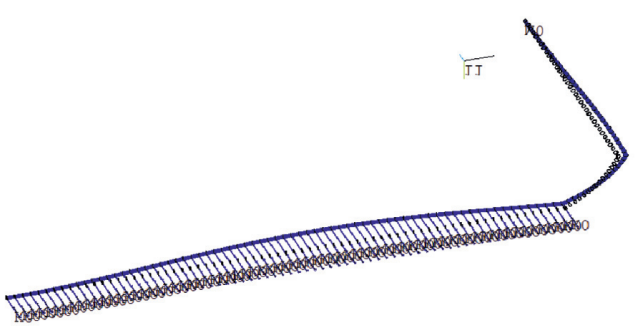

(b)

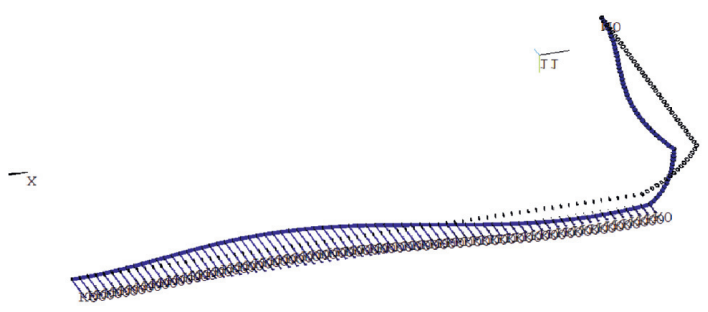

(d)

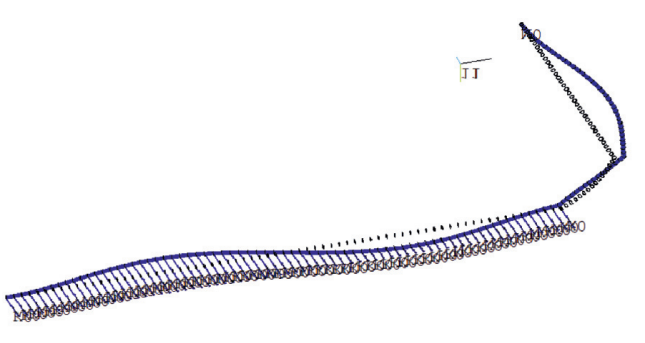

(f)

FIGURE 2: : The first 6 order modal shapes of the SCR: (a) $1^{\text {st }}$ order, (b) $2^{\text {nd }}$ order, (c) $3^{\text {rd }}$ order, (d) $4^{\text {th }}$ order, (e) $5^{\text {th }}$ order, and (f) $6^{\text {th }}$ order.

TABle 2: Parameters of considered oceanic conditions.

\begin{tabular}{|c|c|c|c|c|c|}
\hline Parameters & $\mathrm{OC} 1$ & OC2 & OC3 & OC4 & OC5 \\
\hline Wave height (m) & 5.5 & 5.5 & 5.5 & 5.5 & 5.5 \\
\hline Surface speed $(\mathrm{m} / \mathrm{s})$ & 2.13 & 2.13 & 2.13 & 2.13 & 2.13 \\
\hline Period $(\mathrm{s})$ & 15.4 & 13 & 5.3 & 4.8 & 2.2 \\
\hline
\end{tabular}




$$
\begin{aligned}
\dot{u}_{x}(x, z, t) & =\frac{\delta u_{x}}{\delta t} \\
& =\sum_{n=1}^{\infty} g A_{n} k_{n} \frac{\cosh \left(k_{n}(z+d)\right)}{\cosh \left(k_{n} d\right)} \sin \left(k_{n} x-w_{n} t+\varepsilon_{n}\right), \\
\dot{u}_{z}(x, z, t) & =\frac{\delta u_{z}}{\delta t} \\
& =\sum_{n=1}^{\infty}-g A_{n} k_{n} \frac{\cosh \left(k_{n}(z+d)\right)}{\cosh \left(k_{n} d\right)} \cos \left(k_{n} x-w_{n} t+\varepsilon_{n}\right) .
\end{aligned}
$$

The external loads on the SCR can be determined by the Morison equation:

$$
F=F_{M}+F_{D}+F_{L}
$$

where $F$ is the external force generated by flow; $F_{M}, F_{D}$, and $F_{L}$ are the inertia force, the drag force, and the lift force, respectively, which can be calculated by

$$
\begin{aligned}
F_{M} & =\frac{1}{4} C_{M} \rho \pi D^{2} \dot{u}, \\
F_{D} & =\frac{1}{2} C_{D} \rho D u|u|, \\
F_{L} & =\frac{1}{2} \rho D C_{L} v^{2} \sin \left(\omega_{v} t+\varphi_{v}\right) .
\end{aligned}
$$

Here, $\rho$ is the mass density of the surrounding seawater; $C_{M}, C_{D}$, and $C_{L}$ are the inertia coefficient, the drag coefficient, and the lift force coefficient; $u$ and $\dot{u}$ denote the velocity and acceleration of horizontal water particles; and $D$ is the outside diameter of the pipe.

\section{Pounding Tuned Mass Damper}

3.1. Mechanism of the PTMD. The PTMD is a novel damping device developed on the basis of TMD and impact damper. Figure 3 illustrates the similarities and differences between these three damping devices.
As shown in Figure 3(a), the TMD is composed of an auxiliary mass $\left(m_{2}\right)$ connected to the primary structure $\left(m_{1}\right)$ by a linear spring and a dashpot. The stiffness of the spring $\left(k_{2}\right)$ is determined to tune the TMD to a target frequency. When the primary structure vibrates, the tuned mass $\left(m_{2}\right)$ will provide an antiphase inertial force toward the balancing position, reducing the motion of $m_{1}$ at the targeted frequency.

The impact damper provides damping in another pattern. As shown in Figure 3(b), the impact damper is an added mass $\left(m_{2}\right)$ located on the primary structure $\left(m_{1}\right)$ and restrained by a pair of delimiters. When the major structure oscillates, $m_{2}$ will impact on the delimiter and generate energy dissipation and motion transfer, providing vibration mitigation to $m_{1}$.

The proposed PTMD is a combination of the TMD and the impact damper, and consequently, it has two vibration control mechanisms: (1) if the motion of the major structure is small, the tuned mass moves within the two delimiters and works as a TMD; (2) if the motion of the primary structure is large, the tuned mass will impact on the delimiters and work as an impact damper.

Based on the concept of the PTMD, a clutch-type PTMD (Figure 4) has been developed for the pipeline structures, such as an SCR. The PTMD consists of an L-shaped cantilever beam with a tip mass and a hoop covered with viscoelastic material. The $\mathrm{L}$-shaped beam can be regarded as the spring $\left(k_{2}\right)$ of Figure 3(c). Its geometry shall be determined in order to provide a specific stiffness to make sure the auxiliary mass is tuned to the target frequency. The steel hoop functions as the delimiter of Figure 3(c). When the riser vibrates, the L-shaped beam will bend and impact on the hoop, dissipating energy.

3.2. Simulation of PTMD. The governing equation of a single degree of freedom (DOF) structure equipped with a PTMD is as follows:

$$
\left[\begin{array}{l}
m_{1} \\
m_{2}
\end{array}\right]\left\{\ddot{x}_{1} \ddot{x}_{2}\right\}+\left[\begin{array}{cc}
c_{1} & 0 \\
0 & 0
\end{array}\right]\left\{\begin{array}{c}
\dot{x}_{1} \\
\dot{x}_{2}
\end{array}\right\}+\left[\begin{array}{cc}
k_{1}+k_{2} & -k_{2} \\
-k_{2} & k_{2}
\end{array}\right]\left\{\begin{array}{l}
x_{1} \\
x_{2}
\end{array}\right\}=\left\{\begin{array}{c}
F_{1} \\
F_{2}
\end{array}\right\}+\left[\begin{array}{c}
1 \\
-1
\end{array}\right] F_{P}
$$

where $m_{1}, c_{1}$, and $k_{1}$ are the mass, damping, and stiffness of the primary structure, respectively; $m_{2}$ and $k_{2}$ are the mass and stiffness of the PTMD. $\ddot{x}_{1}, \dot{x}_{1}$, and $x_{1}$ denote the acceleration, velocity, and displacement of the primary structure, while $\ddot{x}_{2}, \dot{x}_{2}$, and $x_{2}$ correspond to those quantities of the damper. In (4), $F_{1}$ and $F_{2}$ are external loading force added on the structure and the PTMD; $F_{P}$ is the pounding force computed by the following equation.

$$
F_{P}= \begin{cases}\beta \delta^{1.5}+c \dot{\delta}, & \dot{\delta}>0, \\ \beta \delta^{1.5}, & \dot{\delta}<0\end{cases}
$$

Here, $F_{P}$ denotes the pounding force, which is determined by the deformation of the viscoelastic delimiter; $\delta$, the velocity of the deformation; $\dot{\delta}$, the pounding stiffness; $\beta$; and the pounding damping, depending on the material property, $c$. The impact damping $c$ is determined by

$$
c=2 \xi \sqrt{\beta \delta^{0.5} \frac{m_{1} m_{2}}{m_{1}+m_{2}}}
$$

where $m_{1}$ and $m_{2}$ are the two colliding bodies and $\xi$ is the pounding damping ratio. 


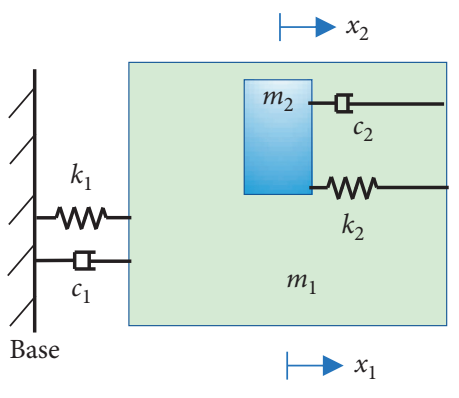

(a)

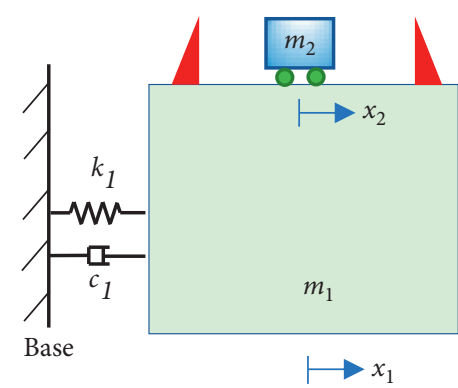

(b)

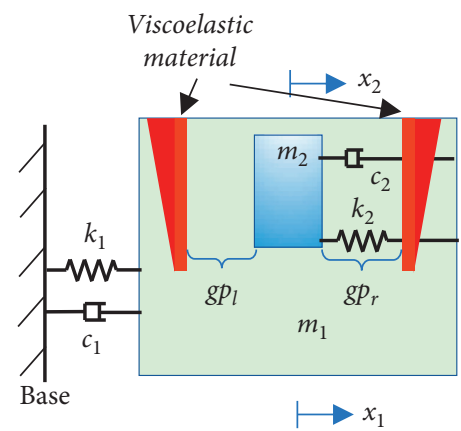

(c)

Figure 3: : Schematics of (a) TMD, (b) impact damper, and (c) PTMD.

$$
\xi=\frac{9 \sqrt{5}}{2} \frac{1-e^{2}}{e(e(9 \pi-16)+16)},
$$

where $e$ is the restitution coefficient of the viscoelastic material. It can be obtained by dropping a sphere on the viscoelastic material, observing the initial height $h_{0}$ and rebound height $h_{1}$.

$$
e=\sqrt{\frac{h_{1}}{h_{0}}}
$$

In previous studies, the motion equation of the PTMD damping system has been established in MATLAB\Simulink environment. The default ordinary equation solver of Simulink (ODE45) is selected to solve the equations. This simulation method has been proven accurate and efficient for structures with limited DOFs. However, the SCR studied in this paper consists of over 600 DOFs, coupled with complicated soil-structure interaction, making it impossible to compute the dynamic response in MATLAB. Therefore, in this study, the aforementioned equations are simulated in ANSYS using the embedded APDL language. A single DOF structure controlled by a PTMD is simulated to verify the ANSYS model. The response of the single DOF structure and the pounding force is presented in Figures 5 and 6. These curves are very close to the results computed by MAT$\mathrm{LAB} \backslash$ Simulink, indicating that the pounding process can be accurately studied by ANSYS.

\section{Vibration Control Performance}

In order to investigate the vibration control effectiveness of the proposed PTMD, an SCR equipped with a PTMD of 5\% mass ratio is simulated in ANSYS. Five oceanic conditions (listed in Table 2) were considered. The vibration reduction ratio is defined as follows to further quantify the oscillation mitigation performance:

$$
\eta=\frac{S_{\text {noctrl }}-S_{P T M D}}{S_{\text {noctrl }}} \times 100 \% .
$$

Here, $S_{\text {noctrl }}$ and $S_{P T M D}$ are the responses of the SCR without and with a PTMD. In (12), $S$ denotes a wide selection of dynamic responses, including displacement, acceleration, moment, or stress.

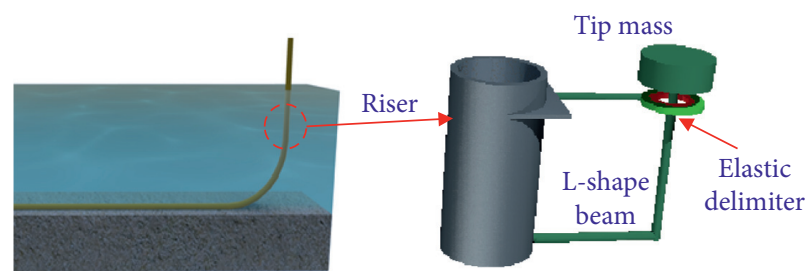

Figure 4: : The PTMD designed for an SCR.

4.1. Vibration Control Performance under 5 Oceanic Conditions. In this section, the SCR with and without the PTMD are subjected to 5 oceanic conditions. Table 3 summarizes the maximum response and reduction ratio, while Figures 7 to 11 demonstrate the displacement and acceleration in the time domain. The blue dashed line denotes the response without control, while the solid red line corresponds to the response with the PTMD. It can be seen that the amplitude varies under different oceanic conditions. The largest displacement took place under OC 4. This is because the wave frequency of OC $4(0.20 \mathrm{~Hz})$ is close to the $1^{\text {st }}$ order frequency of the SCR $(0.18 \mathrm{~Hz})$.

Even though the uncontrolled response of the SCR varied significantly under different oceanic conditions (displacement varied from $0.29 \mathrm{~m}$ to $8.4 \mathrm{~m}$; acceleration varied from $22.07 \mathrm{~m} / \mathrm{s}^{2}$ to $52.00 \mathrm{~m} / \mathrm{s}^{2}$ ), and the PTMD demonstrated satisfying vibration control performance under all these five oceanic conditions. In OC2, the displacement was suppressed by $73.81 \%$, which is also the highest reduction ratio of the five OCs. Even though the reduction ratios in other OCs were not as large as in OC2, the least reduction ratio was also over $40 \%$, demonstrating the effectiveness of the PTMD. In terms of acceleration, the maximum reduction ratio was $52.0 \%$ and the minimum reduction ratio was also over $22 \%$. It also implied that the PTMD was very effective in reducing the acceleration.

4.2. Influence of Mass Ratio. The mass ratio is a key parameter of the dynamic absorbers. The increase in the mass ratio will not only cause the frequency of the structure to shift but also improve the vibration control effectiveness. In this section, PTMDs with a mass ratio of $1 \%$ to $5 \%$ is attached to the SCR. Figure 12 illustrates the displacement of 


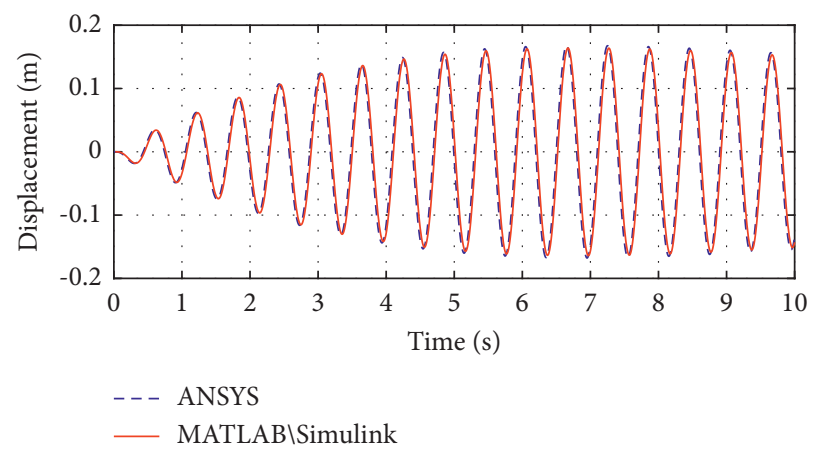

FIgURE 5: : Displacement of a single DOF structure controlled by PTMD.

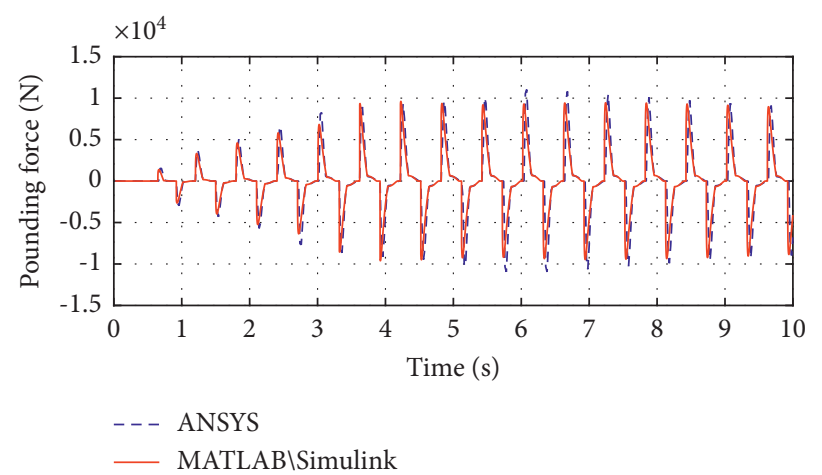

FIgUre 6: : Pounding force of the single DOF structure controlled by PTMD.

TABLE 3: : Vibration control performance of the PTMD.

\begin{tabular}{|c|c|c|c|c|c|}
\hline \multirow{2}{*}{ OC } & \multirow{2}{*}{ SCR } & \multicolumn{2}{|c|}{ Displacement } & \multicolumn{2}{|c|}{ Acceleration } \\
\hline & & Response (m) & Reduction ratio (\%) & Response $\left(\mathrm{m} / \mathrm{s}^{2}\right)$ & Reduction ratio (\%) \\
\hline \multirow{2}{*}{ OC 1} & No control & 6.10 & - & 7.43 & - \\
\hline & PTMD & 3.14 & 48.52 & 3.64 & 51.01 \\
\hline \multirow{2}{*}{ OC 2} & No control & 1.92 & - & 7.47 & - \\
\hline & PTMD & 0.68 & 73.81 & 3.85 & 48.46 \\
\hline \multirow{2}{*}{ OC 3} & No control & 1.92 & - & 9.03 & - \\
\hline & PTMD & 1.09 & 43.22 & 5.88 & 34.88 \\
\hline \multirow{2}{*}{ OC 4} & No control & 8.4 & - & 13.27 & - \\
\hline & PTMD & 3.11 & 62.98 & 6.37 & 52.00 \\
\hline \multirow{2}{*}{ OC 5} & No control & 0.29 & - & 2.91 & - \\
\hline & PTMD & 0.174 & 40.00 & 2.27 & 22.07 \\
\hline
\end{tabular}

the SCR equipped with these PTMDs and the corresponding reduction ratio. Table 4 summarizes the reduction ratio corresponding to OC4. The reduction ratio of other oceanic conditions is shown in Figures 13 and 14.

It can be seen from these figures and tables that the reduction ratio was increased with the increase in mass ratio. When the mass ratio is below $4 \%$, an increase in mass ratio will significantly improve the vibration reduction ratio. However, when the mass ratio is larger than $4 \%$, further increase in mass ratio can only slightly enlarge the reduction ratio. Considering the financial cost, a mass ratio of $3 \%$ is recommended for the practical application of the PTMD.
4.3. Influence of Location of the PTMD. Another parameter to be determined for engineering practice is the place to install the PTMD. In this study, the PTMD is located at node74, node 88 , node 100 , and node 115 as shown in Figure 15. The response of the SCR is compared in Figures 16 and 17.

It is obvious that the highest vibration control efficiency is achieved, while the PTMD is located at node 74 . In this case, the displacement of node 74 , node 88 , node 100 , and node 115 are mitigated by $74.5 \%, 74.2 \%, 77.6 \%$, and $72.5 \%$, respectively. Besides, the vibration reduction ratio of the acceleration also reaches $57.6 \%, 65.4 \%, 26.6 \%$, and $25.6 \%$. On the other hand, however, the vibration is 


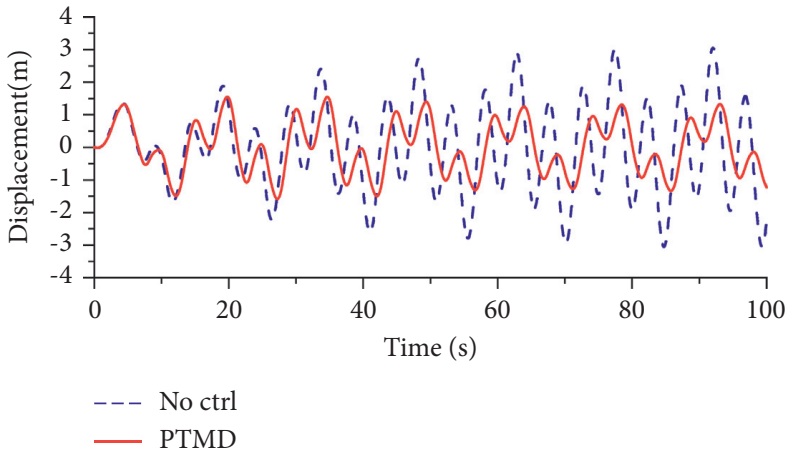

(a)

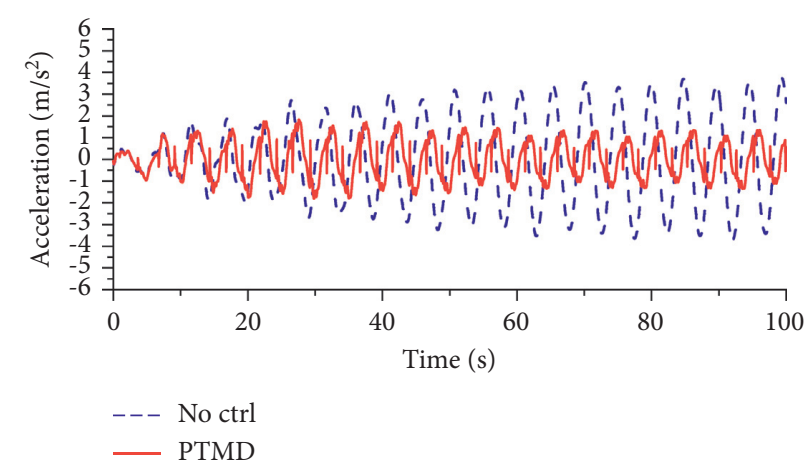

(b)

FIGURE 7: : Maximum response of the SCR under OC1: (a) displacement and (b) acceleration.

TABLE 4: : Vibration control performance of the PTMD with different mass ratios under OC 4.

\begin{tabular}{lcccc}
\hline \multirow{2}{*}{ Mass ratio (\%) } & \multicolumn{2}{c}{ Displacement } & \multicolumn{2}{c}{ Acceleration } \\
& Response $(\mathrm{m})$ & Reduction ratio (\%) & Response $\left(\mathrm{m} / \mathrm{s}^{2}\right)$ & 13.15 \\
Reduction ratio $(\%)$ \\
\hline 1 & 8.08 & 5.3 & 11.96 & 2.0 \\
3 & 6.46 & 24.3 & 9.60 & 28.5 \\
4 & 4.90 & 42.6 & 7.63 & 43.1 \\
5 & 3.80 & 53.5 & 6.37 & 52.0 \\
\hline
\end{tabular}

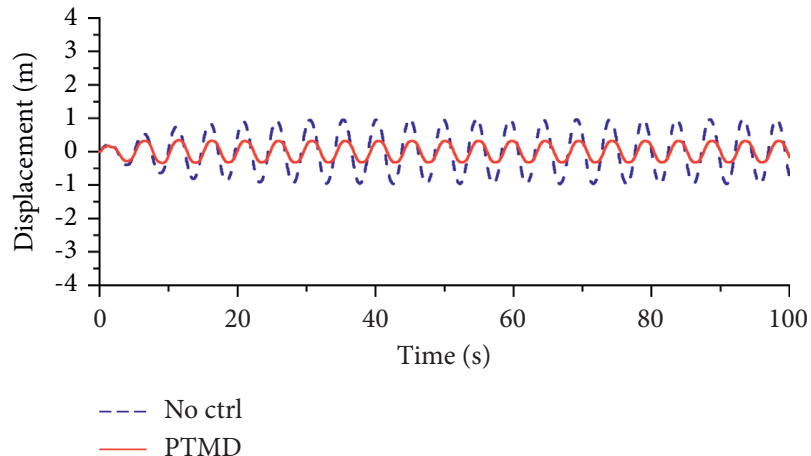

(a)

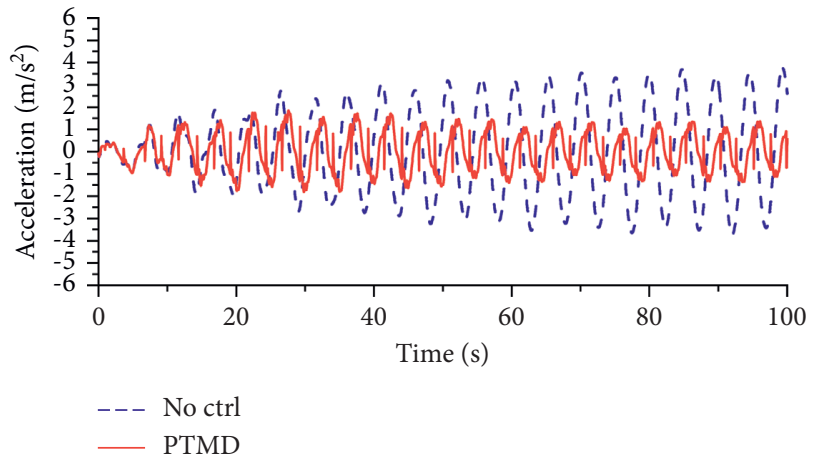

(b)

FIgURE 8: : Maximum response of the SCR under OC2: (a) displacement and (b) acceleration.

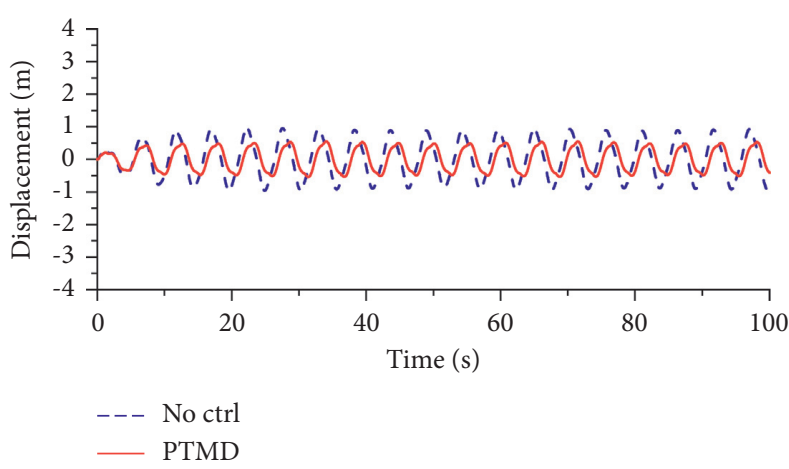

(a)

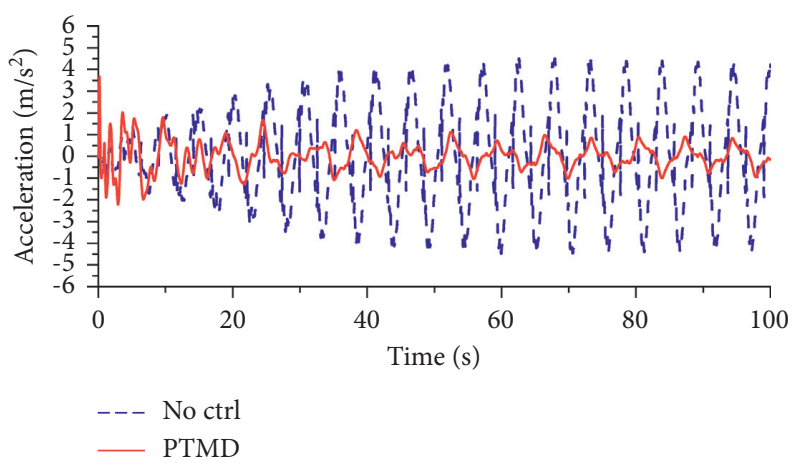

(b)

FIgURE 9: : Maximum response of the SCR under OC3: (a) displacement and (b) acceleration. 


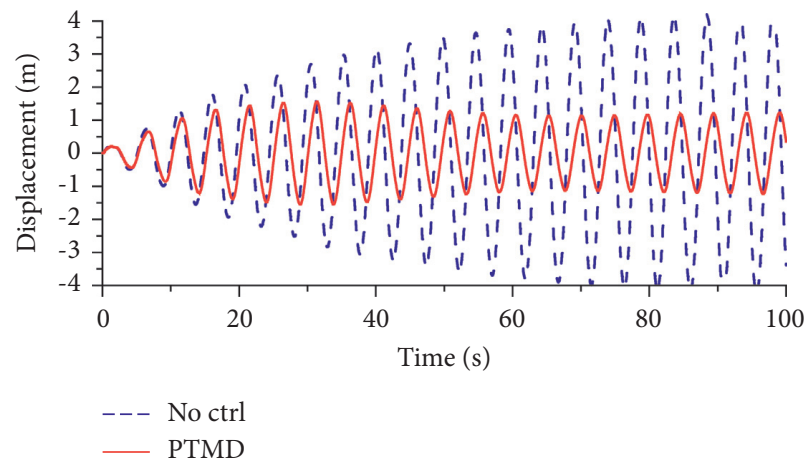

(a)

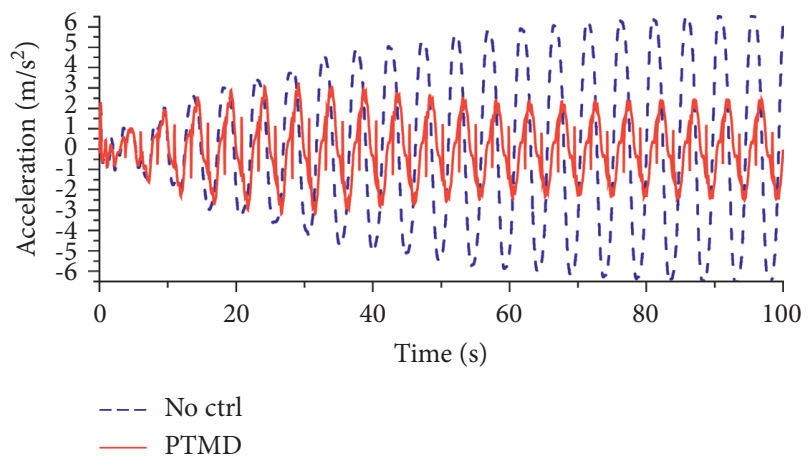

(b)

FIGURE 10: : Maximum response of the SCR under OC4: (a) displacement and (b) acceleration.

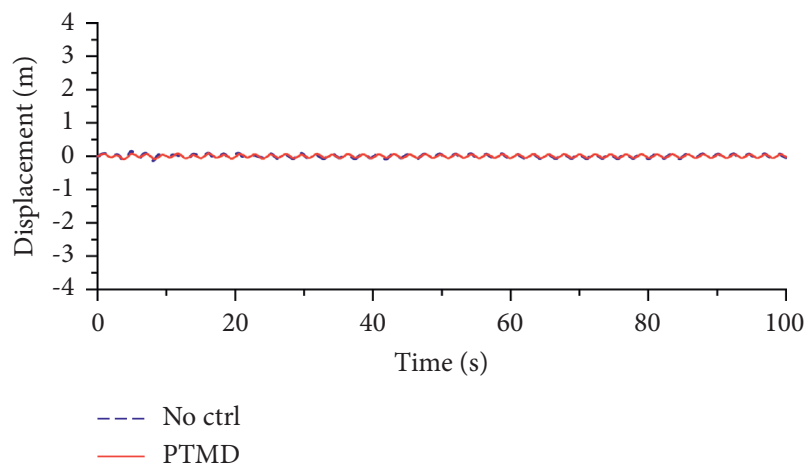

(a)

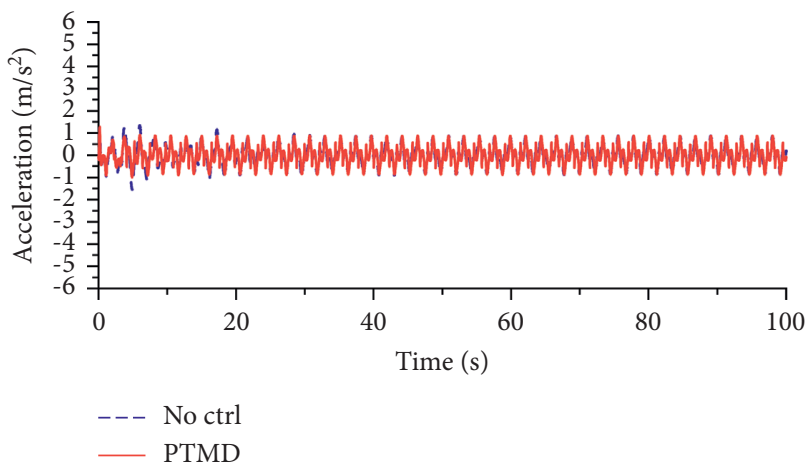

(b)

FIGURE 11: : Maximum response of the SCR under OC5: (a) displacement and (b) acceleration.

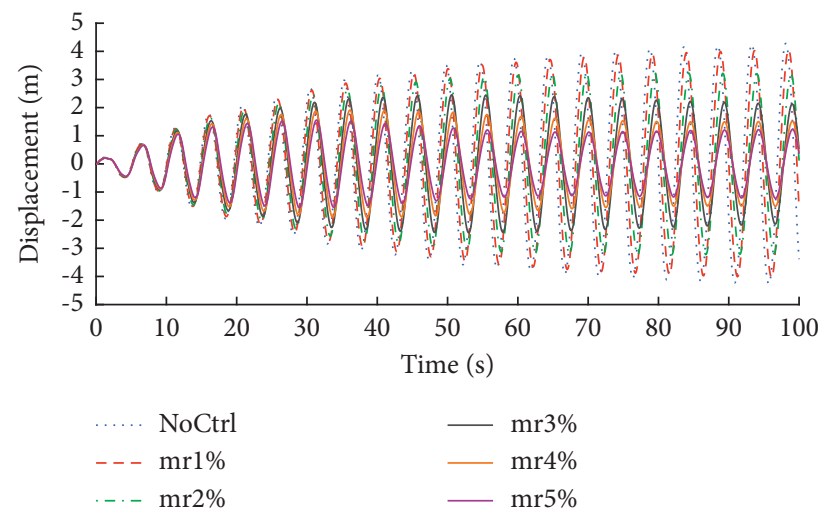

FIgURE 12: : Time history of the displacement of the SCR implemented with PTMD of mass ratio from $1 \%$ to $5 \%$ at oceanic condition 4.

hardly reduced if the PTMD is located at node 115 . The vibration reduction ratio is around $3 \%$ for both displacement and acceleration, as depicted in Figures 16 and 17. The main reason is that the motion of node 115 is very small compared with node 74 or node 88 . Therefore, in practical engineering, it is recommended that the PTMD be installed at the location where the largest displacement takes place. 


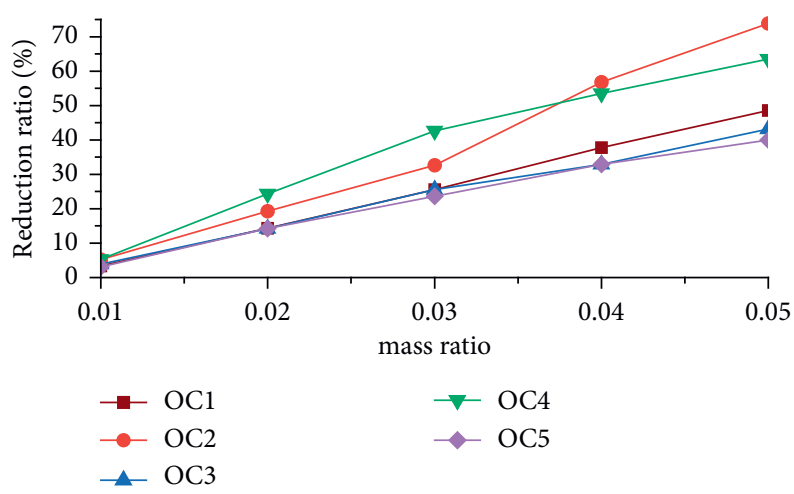

Figure 13: : Displacement reduction ratio of PTMDs of mass ratio from $1 \%$ to $5 \%$.

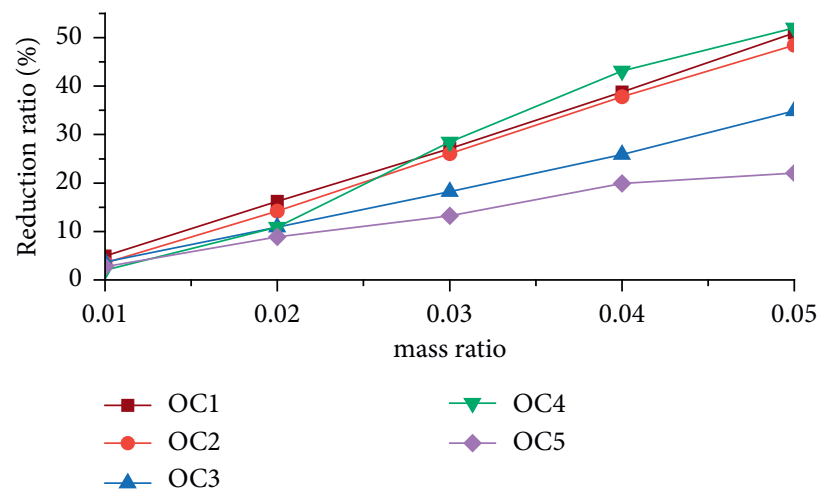

FIGURE 14: : Acceleration reduction ratio of PTMDs of mass ratio from $1 \%$ to $5 \%$.

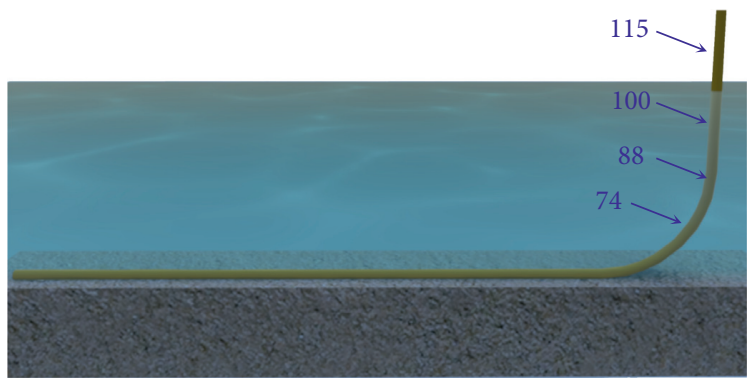

FIGURE 15: : Position of the PTMD.

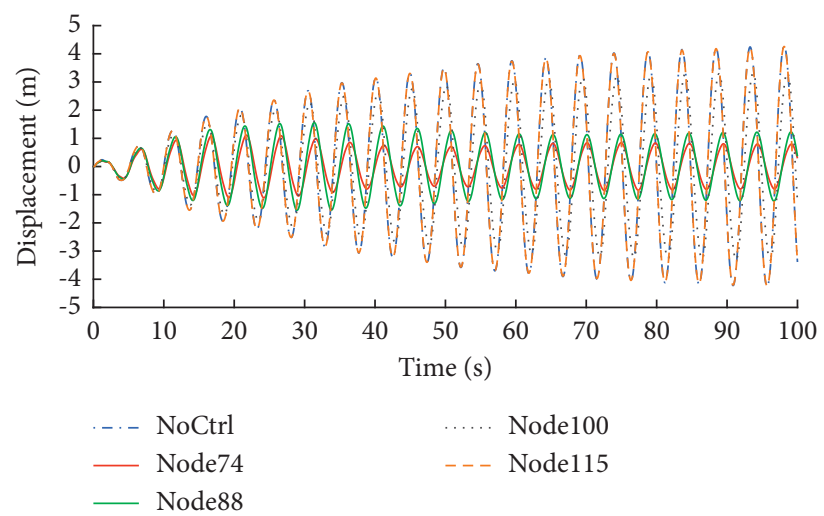

FIGURE 16: : Time history of the displacement of the SCR implemented with PTMD installed at different places. 


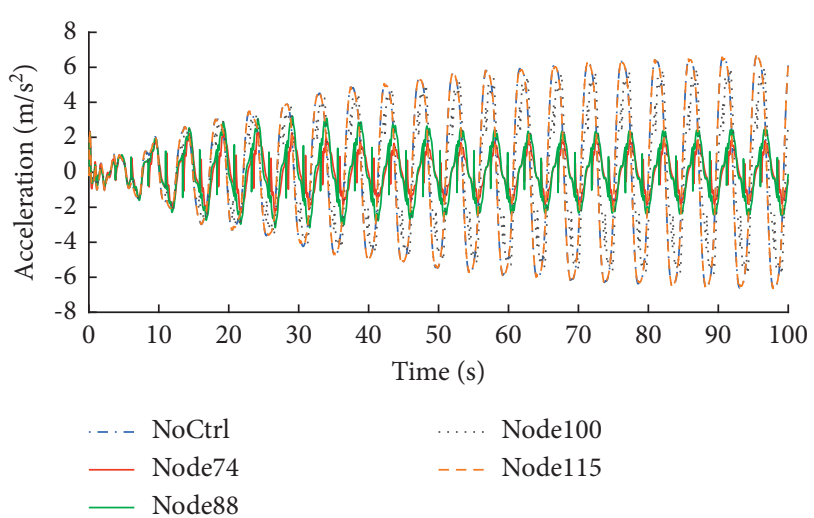

FIgURE 17: : Time history of the acceleration of the SCR implemented with PTMD installed at different places.

\section{Conclusions}

This paper discussed the vibration control efficiency of PTMD against the flow-induced vibration of an SCR. The SCR was modeled by PIPE 59 elements in ANSYS, and the interaction between the pipes and the seabed soil was simulated by $p-y$ curves. The PTMD was also modeled in ANSYS using the APDL language. Five typical oceanic conditions were considered when investigating the vibration control efficiency and the influence of mass ratio and the location of the PTMD. The main conclusions are as follows:

(1) The PTMD provided sufficient damping for the SCR. Under oceanic condition 2, the displacement was mitigated by $73.8 \%$. Under other oceanic conditions, the vibration reduction ratio of displacement was all above $40 \%$, indicating the vibration control efficiency of the PTMD is robust under different situations.

(2) The vibration control efficiency of displacement was higher than that of acceleration. This is similar to other types of dynamic absorbers.

(3) The vibration reduction effectiveness was upgraded with the increase in mass ratio. However, considering the financial cost and the limited installation space, a mass ratio of $3 \%$ is still recommended for engineering practice.

(4) The location of the PTMD also demonstrated a great influence on the vibration control effectiveness. In this study, the optimal position of the PTMD is at node 74 , which is near the touchdown point of the pipe.

\section{Data Availability}

The data used to support the findings of this study are available from the corresponding author upon request.

\section{Conflicts of Interest}

The authors declare that there are no conflicts of interest regarding the publication of this paper.

\section{Acknowledgments}

This research was funded by the National Natural Science Foundation of China (51808092); the United Navigation Foundation of Liaoning Province (2020-HYLH-48); the National Key Research and Development Program of China (2021YFB2601102); and the open fund of State Key Laboratory of Coastal and Offshore Engineering (DUT-LP2122).

\section{References}

[1] P. Wang, Y. Xu, X. Zhang, R. Xi, and X. Du, "A substructure method for seismic responses of offshore wind turbine considering nonlinear pile-soil dynamic interaction," Soil Dynamics and Earthquake Engineering, vol. 144, Article ID 106684, 2021.

[2] J. P. R. Cortina, F. J. M. de Sousa, and L. V. S. Sagrilo, "Neural networks applied to the wave-induced fatigue analysis of steel risers," Mathematical Problems in Engineering, vol. 2018, Article ID 2719682, 16 pages, 2018.

[3] Y. Wang, Z. Wu, and X. Zhang, "Research on bifurcation response for vortex-induced vibration of top tension riser in shear flow," Mathematical Problems in Engineering, vol. 2019, Article ID 1564194, 13 pages, 2019.

[4] F. Wang, W. Xiao, Y. Yao, Q. Liu, and C. Li, "An analytical procedure to predict transverse vibration response of jack-up riser under the random wave load," Shock and Vibration, vol. 2020, Article ID 5072989, 9 pages, 2020.

[5] T. Ren, Y.-w. Xu, J. Wang et al., "An experiment study of vortex induced vibration of a steel catenary riser under steady current," Journal of Hydrodynamics, vol. 32, no. 5, pp. 834-844, 2020.

[6] H. Zhu, Y. Gao, and H. Zhao, "Experimental investigation on the flow-induced vibration of a free-hanging flexible riser by internal unstable hydrodynamic slug flow," Ocean Engineering, vol. 164, pp. 488-507, 2018.

[7] J. Lian, X. Yan, F. Liu, and J. Zhang, "Analysis on flow induced motion of cylinders with different cross sections and the potential capacity of energy transference from the flow," Shock and Vibration, vol. 2017, pp. 1-19, 2017.

[8] J. Zhang, Y. Zeng, Y. Tang, W. Guo, and Z. Wang, "Numerical and experimental research on the effect of platform heave motion on vortex-induced vibration of deep sea top-tensioned riser," Shock and Vibration, vol. 2021, Article ID 8866051, 13 pages, 2021.

[9] M. Zhang, S. Fu, C. Liu, H. Ren, and Y. Xu, "Experimental investigation on vortex-induced force of a Steel Catenary Riser under in-plane vessel motion," Marine Structures, vol. 78, Article ID 102882, 2021.

[10] J. C. Lecordier, L. Hamma, and P. Paranthoen, "The control of vortex shedding behind heated circular cylinders at low Reynolds numbers," Experiments in Fluids, vol. 10, no. 4, pp. 224-229, 1991.

[11] G. Artana, R. Sosa, E. Moreau, and G. Touchard, "Control of the near-wake flow around a circular cylinder with electrohydrodynamic actuators," Experiments in Fluids, vol. 35, no. 6, pp. 580-588, 2003.

[12] W. Wang, B. Song, Z. Mao, W. Tian, and T. Zhang, "Numerical investigation on VIV suppression of marine risers attached with rotating wings," Chuan Bo Li Xue/Journal of Ship Mechanics, vol. 25, pp. 29-36, 2021.

[13] Z. Zhao, S. Lin, D. Zhu, and G. Wen, "Vibration control of a riser-vessel system subject to input backlash and extraneous 
disturbances," IEEE Transactions on Circuits and Systems II: Express Briefs, vol. 67, no. 3, pp. 516-520, 2020.

[14] Y. Liu, Q. Zhang, and Y. Fu, "Vibration suppression of a flexible riser by boundary iterative learning control," IEEE Access, vol. 7, Article ID 130269, 2019.

[15] S. Zhang, X. He, and C. Yang, "Vibration control of a flexible marine riser with joint angle constraint," Nonlinear Dynamics, vol. 87, no. 1, pp. 617-632, 2017.

[16] Y. Liu and F. Guo, "Output feedback boundary control of a flexible marine riser system," Journal of Vibration and Control, vol. 24, no. 16, pp. 3617-3630, 2017.

[17] F. Guo, Y. Liu, Z. Zhao, and F. Luo, "Adaptive vibration control of a flexible marine riser via the backstepping technique and disturbance adaptation," Transactions of the Institute of Measurement and Control, vol. 40, no. 5, pp. 1407-1416, 2018.

[18] Q. Liu, W. Hao, C. Li, W. Miao, and Q. Ding, "Numerical simulation on the forced oscillation of rigid riser with helical strakes in different section shapes," Ocean Engineering, vol. 190, 2019.

[19] P. Li, L. Liu, Z. Dong, F. Wang, and H. Guo, "Investigation on the spoiler vibration suppression mechanism of discrete helical strakes of deep-sea riser undergoing vortex-induced vibration," International Journal of Mechanical Sciences, vol. 172, 2020.

[20] Z. Gao, M. Efthymiou, L. Cheng, T. Zhou, M. Minguez, and W. Zhao, "Towards a model of hydrodynamic damping for a circular cylinder with helical strakes at low KC," Marine Structures, vol. 78, 2021.

[21] N. Kumar, V. Kumar Varma Kolahalam, M. Kantharaj, and S. Manda, "Suppression of vortex-induced vibrations using flexible shrouding-An experimental study," Journal of Fluids and Structures, vol. 81, pp. 479-491, 2018.

[22] M. M. Cicolin and G. R. S. Assi, "Experiments with flexible shrouds to reduce the vortex-induced vibration of a cylinder with low mass and damping," Applied Ocean Research, vol. 65, pp. 290-301, 2017.

[23] G. R. S. Assi, P. W. Bearman, and M. A. Tognarelli, "On the stability of a free-to-rotate short-tail fairing and a splitter plate as suppressors of vortex-induced vibration," Ocean Engineering, vol. 92, pp. 234-244, 2014.

[24] H. Zhu, Z. Liao, Y. Gao, and Y. Zhao, "Numerical evaluation of the suppression effect of a free-to-rotate triangular fairing on the vortex-induced vibration of a circular cylinder," $A p$ plied Mathematical Modelling, vol. 52, pp. 709-730, 2017.

[25] M. Lou, W.-g. Wu, and P. Chen, "Experimental study on vortex induced vibration of risers with fairing considering wake interference," International Journal of Naval Architecture and Ocean Engineering, vol. 9, no. 2, pp. 127-134, 2017.

[26] X. Sun, C. Steve Suh, C. Sun, and B. Yu, "Vortex-induced vibration of a flexible splitter plate attached to a square cylinder in laminar flow," Journal of Fluids and Structures, vol. 101, 2021.

[27] S. Liang, J. Wang, B. Xu, W. Wu, and K. Lin, "Vortex-induced vibration and structure instability for a circular cylinder with flexible splitter plates," Journal of Wind Engineering and Industrial Aerodynamics, vol. 174, pp. 200-209, 2018.

[28] H. Zhu, G. Li, and J. Wang, "Flow-induced vibration of a circular cylinder with splitter plates placed upstream and downstream individually and simultaneously," Applied Ocean Research, vol. 97, 2020.

[29] S. Liang, J. Wang, and Z. Hu, "VIV and galloping response of a circular cylinder with rigid detached splitter plates," Ocean Engineering, vol. 162, pp. 176-186, 2018.
[30] H. Matin Nikoo, K. Bi, and H. Hao, "Passive vibration control of cylindrical offshore components using pipe-in-pipe (PIP) concept: an analytical study," Ocean Engineering, vol. 142, pp. 39-50, 2017.

[31] K. Bi and H. Hao, "Using pipe-in-pipe systems for subsea pipeline vibration control," Engineering Structures, vol. 109, pp. 75-84, 2016.

[32] H. Matin Nikoo, K. Bi, and H. Hao, "Textured pipe-in-pipe system: a compound passive technique for vortex-induced vibration control," Applied Ocean Research, vol. 95, 2020.

[33] W. Wang, Z. Yang, X. Hua, Z. Chen, X. Wang, and G. Song, "Evaluation of a pendulum pounding tuned mass damper for seismic control of structures," Engineering Structures, vol. 228, Article ID 111554, 2021.

[34] P. Zhang, L. Li, D. Patil et al., "Parametric study of pounding tuned mass damper for subsea jumpers," Smart Materials and Structures, vol. 25, Article ID 15028, 2015.

[35] H. Li, P. Zhang, G. Song, D. Patil, and Y. Mo, "Robustness study of the pounding tuned mass damper for vibration control of subsea jumpers," Smart Materials and Structures, vol. 24, 2015.

[36] J. Jiang, P. Zhang, D. Patil, H. Li, and G. Song, "Experimental studies on the effectiveness and robustness of a pounding tuned mass damper for vibration suppression of a submerged cylindrical pipe," Structural Control and Health Monitoring, vol. 24, Article ID e2027, 2017.

[37] G. B. Song, P. Zhang, L. Y. Li, M. Singla, and D. Patil, "Vibration Control of a Pipeline Structure Using Pounding Tuned Mass Damper," Journal of Engineering Mechanics, vol. 142, 2016.

[38] D. Patil, A. Kalia, G. B. Song, and M. Lara, "An Innovative Damper for Subsea Pipeline and Jumper Vibration Controls," in Proceedings of the Offshore Technology Conference, Houston, TX, USA, May 2017.

[39] W. Wang, X. Wang, X. Hua, G. Song, and Z. Chen, "Vibration control of vortex-induced vibrations of a bridge deck by a single-side pounding tuned mass damper," Engineering Structures, vol. 173, pp. 61-75, 2018.

[40] L. Li, G. Song, M. Singla, and Y. Mo, "Vibration control of a traffic signal pole using a pounding tuned mass damper with viscoelastic materials (II): experimental verification," Journal of Vibration and Control, vol. 21, no. 4, pp. 670-675, 2013.

[41] N. Zhao, G. Huang, R. Liu, P. Zhang, C. Lu, and G. Song, "Novel hidden pounding tuned mass damper for vibration control of a cantilevered traffic signal structure," Journal of Engineering Mechanics, vol. 146, 2020.

[42] J. Tan, S. Michael Ho, P. Zhang, and J. Jiang, "Experimental study on vibration control of suspended piping system by single-sided pounding tuned mass damper," Applied Sciences, vol. 9, no. 2, p. 285, 2019.

[43] H. Guo, W. Dong, and M. Lou, "Vortex-induced vibration testing and fatigue life analysis of practical risers conveying fluid," Periodical of ocean university of china, vol. 38, pp. 503-507, 2008.

[44] G. Zhang, P. Wang, M. Zhao, X. Du, and X. Zhao, "Seismic structure-water-sediment-rock interaction model and its application to immersed tunnel analysis under obliquely incident earthquake," Tunnelling and Underground Space Technology, vol. 109, Article ID 103758, 2021.

[45] C. Cui, K. Meng, C. Xu, Z. Liang, H. Li, and H. Pei, "Analytical solution for longitudinal vibration of a floating pile in saturated porous media based on a fictitious saturated soil pile model," Computers and Geotechnics, vol. 131, Article ID 103942, 2021. 
[46] K. Meng, C. Cui, Z. Liang, H. Li, and H. Pei, "A new approach for longitudinal vibration of a large-diameter floating pipe in visco-elastic soil considering the three-dimensional wave effects," Computers and Geotechnics, vol. 128, Article ID 103840, 2021.

[47] Y. Yuan, M. Zheng, H. Xue, and W. Tang, "Nonlinear riserseabed interaction response among touchdown zone of a steel catenary riser in consideration of vortex-induced vibration," Ocean Engineering, vol. 227, Article ID 108891, 2021.

[48] C. P. Aubeny, G. Biscontin, and G. J. Zhang, Seafloor interaction with steel catenary risers, Final Project Report to Minerals Management Service, Offshore Technology Research Centre Industry Consortium, Texas A\&M University, College Station, OTRC Library, Houston, TX, USA, 2006.

[49] A. Fournier and W. T. Reeves, "A Simple Model of Ocean Waves," Computer Graphics, vol. 20, 1986. 